\title{
Top-Down Restoration of Speech in Cochlear-Implant Users
}

\author{
Pranesh Bhargava ${ }^{1,2, a)}$, Etienne Gaudrain ${ }^{1,2}$, Deniz Başkent ${ }^{1,2}$ \\ 1 University of Groningen, University Medical Center Groningen, Department of Otorhinolaryngology/Head \\ and Neck Surgery, The Netherlands \\ 2 University of Groningen, Graduate School of Medical Sciences, Research School of Behavioral and Cognitive \\ Neurosciences, The Netherlands \\ E-mails: p.bhargava@umcg.nl, e.p.c.gaudrain@umcg.nl, d.baskent@umcg.nl
}

\begin{abstract}
In noisy listening conditions, intelligibility of degraded speech can be enhanced by top-down restoration. Cochlear implant (CI) users have difficulty understanding speech in noisy environments. This could partially be due to reduced top-down restoration of speech, which may be related to the changes that the electrical stimulation imposes on the bottom-up cues. We tested this hypothesis using the phonemic restoration $(\mathrm{PhR})$ paradigm in which speech interrupted with periodic silent intervals is perceived illusorily continuous (continuity illusion or CoI) and becomes more intelligible (PhR benefit) when the interruptions are filled with noise bursts. Using meaningful sentences, both $\mathrm{CoI}$ and PhR benefit were measured in CI users, and compared with those of normal-hearing $(\mathrm{NH})$ listeners presented with normal speech and 8-channel noise-band vocoded speech, acoustically simulating CIs. CI users showed different patterns in both PhR benefit and CoI, compared to $\mathrm{NH}$ results with or without the noise-band vocoding. However, they were able to use top-down restoration under certain test conditions. This observation supports the idea that changes in bottom-up cues can impose changes to the top-down processes needed to enhance intelligibility of degraded speech. The knowledge that CI users seem to be able to do restoration under the right circumstances could be exploited in patient rehabilitation and product development.
\end{abstract}

Keywords: Cochlear implants, speech perception, top-down restoration, phonemic restoration, continuity illusion.

\footnotetext{
a) Author to whom correspondence should be addressed: p.bhargava@umcg.nl
} 


\section{INTRODUCTION}

In real-life listening conditions, background noise often masks the target sounds of interest. Using bottom-up signal cues that are audible through the masking noise and top-down cognitive mechanisms, normal-hearing $(\mathrm{NH})$ listeners are capable of perceptually restoring these degraded sounds. Two aspects of such restoration are continuity illusion (CoI) and phonemic restoration (PhR). CoI stems from the fact that, perhaps relying on Gestalt principles, the auditory system tends to perceive parts of a signal belonging to one speech stream, instead of as individual, segmented utterances (Bregman, 1990; Shinn-Cunningham, 2008). As a result of this tendency, even if a signal is interrupted with silence, when the interruption is filled with noise, the signal may be perceived to be illusorily continuous (Carlyon et al., 2002; Heinrich et al., 2008; King, 2007; Miller and Licklider, 1950; Riecke et al., 2012, 2009; Thurlow, 1957; Thurlow and Elfner, 1959; Warren et al., 1972). PhR is considered to be a special case of top-down restoration. When the interrupted signal is speech, the addition of noise in the silent interruptions increases ambiguity, which likely helps lexical activation of many more word candidates (Srinivasan and Wang, 2005). Under certain conditions, this results in an increase of intelligibility (Bashford and Warren, 1979; Kashino, 2006; Powers and Wilcox, 1977; Verschuure and Brocaar, 1983). PhR can therefore be used as a measure of the top-down restoration of degraded speech (Başkent, 2010; Benard and Başkent, 2013; Saija et al., 2013; Warren and Obusek, 1971).

Literature implies that $\mathrm{CoI}$ and $\mathrm{PhR}$ are associated with each other. Some research suggested $\mathrm{CoI}$ to be a prerequisite to the increased intelligibility observed with $\mathrm{PhR}$, while others suggested that the two are separate mechanisms with partial overlap (Başkent et al., 2009; Repp, 1992; Riecke et al., 2011, 2009; Shahin et al., 2009; Thurlow, 1957; Warren et al., 1994). Incidentally, CoI has been observed in other species as well (Petkov et al., 2003; Sugita, 1997), opening the possibility that it may be a precursor to the linguistic form of topdown completion, i.e., PhR. On the other hand, since animals cannot be tested for PhR, animal studies do not give a direct evidence of the relationship between $\mathrm{CoI}$ and $\mathrm{PhR}$.

$\mathrm{PhR}$ relies on exploiting the information from speech features in the remaining or audible speech segments, and uses various top-down mechanisms such as applying linguistic knowledge, situational or semantic context, and expectations in order to reconstruct the obliterated parts of speech (Bashford and Warren, 1979; Kashino, 2006; Powers and Wilcox, 1977; Shahin et al., 2009; Verschuure and Brocaar, 1983; Warren, 1970). Since PhR involves restoring the obliterated speech using the speech features from the audible speech segments, the quality of these segments is likely to have an effect on the degree of PhR. This idea was indirectly confirmed by studies that showed lack of or reduced phonemic restoration, when bottom-up speech cues were degraded due to hearing loss or cochlear-implant simulations (Başkent, 2012, 2010). The intact portions of the speech must thus provide appropriate bottom-up cues in order to activate the relevant knowledge that will trigger the restoration through top-down mechanisms (Srinivasan and Wang, 2005).

In users of cochlear implants (CIs), the bottom-up cues may be affected by the various limitations of CI processing, e.g., reduction in spectral resolution and temporal fine structure in the signal, as well as possible distortions caused by electrode placement (Başkent and Shannon, 2006, 2005) and electrode-neuron interface (Nelson and Jin, 2004; Qin and Oxenham, 2003). Additionally, front-end processing may also affect the bottom-up speech cues. An example of this is automatic gain control (AGC), which could potentially affect speech envelope due to the attack and release time constants. Many CI users experience difficulty understanding speech in noisy environments, even if they have a good intelligibility of speech in quiet (Fu et al., 1998; Nelson and Jin, 2004; Nelson et al., 2003; Stickney et al., 2004). Some of this difficulty may be caused by reduced top-down restoration due to 
degraded bottom-up speech cues. Using an acoustic simulation of CIs with NH listeners, which would only capture the effect of impoverished spectral and temporal resolution, Başkent (2012) indeed observed poorer PhR. Further, using a simplified simulation of AGC, Başkent et al. (2009) observed that speech envelope fluctuations caused by AGCs can reduce both $\mathrm{CoI}$ and $\mathrm{PhR}$ for a range of time constants that are similar to those used in hearing devices.

In this study, we tested top-down restoration of speech, both in terms of $\mathrm{CoI}$ and $\mathrm{PhR}$, by $\mathrm{CI}$ users, as well as by NH listeners tested with and without an acoustic simulation of CIs. Due to the factors listed above and based on the simulation studies by Başkent et al. (2009) and Başkent (2012), we expected CI users, as well as acoustic simulation conditions, to show reduced or no benefit from $\mathrm{PhR}$. Expectations on CoI, however were not as clear. The reduction in signal quality due to CI speech transmission is perhaps not sufficient by itself to reduce $\mathrm{CoI}$ - if anything, if the filler noise and speech segments have more comparable sound quality, perhaps they are more easily fused, causing a stronger continuity percept. On the other hand, as simulations by Başkent et al. (2009) suggested, some features of front-end processing may work against $\mathrm{CoI}$. If $\mathrm{CoI}$ is indeed a prerequisite stage to $\mathrm{PhR}$, its reduction could further hinder the intelligibility benefit of PhR. Any reductions in restoration of speech by CI users, either in PhR or CoI, are important to identify, as these are key mechanisms of perceptual organization. Further, these could be contributing factors to the CI users' problems of understanding speech in background noise. With such knowledge, interactions of the bottom-up signals with top-down mechanisms can perhaps be better incorporated into the development of new device features or rehabilitation of CI users. For example, tests that can better capture such interactions can be developed for these applications.

TABLE I. Details of CI participants. 'n.a.' denotes that the readings were not available in the patient record.

\begin{tabular}{|l|l|l|l|l|l|l|}
\hline $\begin{array}{l}\text { Subje } \\
\text { ct ID }\end{array}$ & $\begin{array}{l}\text { Gen } \\
\text { der }\end{array}$ & $\begin{array}{l}\text { Age at } \\
\text { the time } \\
\text { of the } \\
\text { experim } \\
\text { ent (yrs.) }\end{array}$ & $\begin{array}{l}\text { Age at } \\
\text { onset of } \\
\text { hearing } \\
\text { loss } \\
\text { (yrs.) }\end{array}$ & $\begin{array}{l}\text { Age when } \\
\text { started using a } \\
\text { hearing aid } \\
\text { (yrs.) }\end{array}$ & $\begin{array}{l}\text { Duration of } \\
\text { CI usage } \\
\text { (yrs. })\end{array}$ & $\begin{array}{l}\text { CI brand (and processor) } \\
\text { AB= Advanced Bionics } \\
\text { CO= Cochlear }\end{array}$ \\
\hline CI 1 & F & 28 & n.a. & n.a. & 10 & CO CI24R CS \\
\hline CI 2 & M & 38 & 3 & 3 & 1.5 & CO CI24RE CA \\
\hline CI 3 & F & 22 & n.a. & n.a. & 9 & CO CI24R CS \\
\hline CI 4 & M & 23 & n.a. & n.a. & 8 & CO CI24R CA \\
\hline CI 5 & F & 65 & 30 & n.a. & 12 & CO CI24R CS \\
\hline CI 6 & M & 52 & 33 & 33 & 7 & CO CI24R CA \\
\hline CI 7 & M & 65 & 61 & n.a. & 3 & CO CI24RE CA \\
\hline CI 8 & F & 62 & 45 & 50 & 3 & CO CI24R CS \\
\hline CI 9 & M & 64 & n.a. & n.a. & 3 & CO CI24R CA \\
\hline CI 10 & F & 57 & n.a. & n.a. & 9 & CO CI24R CA \\
\hline CI 11 & F & 65 & n.a. & n.a. & 4 & CO CI24RE CA \\
\hline CI 12 & M & 35 & 1 & n.a. & 10 & CO CI24R CS \\
\hline CI 13 & M & 55 & 0 & 7 & 4 & AB HiRes 90K Helix \\
\hline
\end{tabular}




\section{MATERIALS AND METHODS}

\section{A. Participants}

Fourteen NH listeners (from 19 to 28 years; average age 23 years; 7 females) and 13 CI users (from 22 to 65 years; average age 49 years; 6 females) took part in the study. All participants were native speakers of the Dutch language. They reported no linguistic disability.

The NH participants had a pure tone hearing threshold average across test frequencies of 0.5 , 1,2 and $4 \mathrm{kHz}$ at the better ear that was lower or equal to $20 \mathrm{~dB}$ HL (Stephens, 1996). These participants were tested once without (NHnorm) and once with the acoustic simulation of CI (NHCI).

The CI users were recruited through the clinic of the Otorhinolaryngology Department, University Medical Center Groningen. The details of the CI participants are provided in Table I. All CI users were monaurally implanted and had more than one year of experience with their CI device prior to the commencement of the experiment. Participants with relatively high $(\geq 70 \%)$ phoneme scores in quiet for monosyllabic (CVC) meaningful words (Bosman, 1989) were selected in order to minimize floor effects (Table II). No significant correlation was found between the phoneme scores and duration of implant usage or age of the participant at the time of experiment.

Prior to the training, the CI users were familiarized with the highest and lowest SNR conditions in order for them to adjust their device to the volume they found most comfortable without affecting the speech intelligibility. This setting was not changed during the training or the main experiment. None of the CI users used an acoustic hearing aid during the experiment.

Written informed consent was provided by all participants before taking part in the experiment. The participants received financial compensation for their participation in the study. The study was approved by the Medical Ethical Review Committee of the University Medical Center Groningen.

\section{B. Stimuli}

Complete, syntactically and grammatically correct and meaningful Dutch sentences with semantically neutral content (without referring to the topics of current affairs, specific issues, etc.), taken from the Vrij University (VU) corpus (Versfeld et al., 2000), were used as the speech stimuli. An example sentence is "Buiten is het donker en koud," meaning "Outside it is dark and cold". The corpus comprises two sets of 39 lists each (one spoken by a male speaker and the other by a female speaker), each list containing 13 sentences, digitally recorded at the sampling rate of $44.1 \mathrm{kHz}$. There are 80 words per list on average.

For the present study, 38 lists uttered by the male speaker were used. Three lists, of which one was used for training and two to measure the VU baseline score, were not included in the main experiment. For NH listeners, two non-overlapping subsets of ten lists were randomly chosen for each of the NHnorm and NHCI conditions. Similarly, for CI users, for each of the two sessions, ten non-overlapping lists were randomly chosen. For each participant, the same set of lists and conditions used for measuring PhR were also used to measure CoI, though the combination of lists and conditions was randomized. 
TABLE II. Free-field pure-tone thresholds of the non-implanted ear for CI participants vis-à-vis their clinical speech scores, measured with CVC words, and experimental VU baseline scores, measured with unprocessed sentences. An empty set symbol $(\varnothing)$ denotes where the participant did not respond up to $100 \mathrm{~dB}$ HL. Parenthesized value shows the threshold for the implanted ear. The 'clinical speech intelligibility score' is the percentage of correctly reported phonemes in meaningful CVC words (measured at $75 \mathrm{~dB}$ SPL). The 'VU baseline intelligibility' was measured with the material used during the experiment. It is the RAU of correctly reported words in meaningful sentences (measured at $60 \mathrm{~dB} \mathrm{~A}$ ).

\begin{tabular}{|c|c|c|c|c|c|c|c|c|}
\hline \multirow{2}{*}{$\begin{array}{l}\text { Subject } \\
\text { ID }\end{array}$} & \multicolumn{6}{|c|}{ Tone thresholds of non-implanted ear (dB HL) } & \multirow{2}{*}{$\begin{array}{l}\text { Clinical speech } \\
\text { intelligibility } \\
\text { score in percent } \\
\text { correct }\end{array}$} & \multirow{2}{*}{$\begin{array}{c}\text { VU baseline } \\
\text { intelligibility in } \\
\text { RAU }\end{array}$} \\
\hline & $125 \mathrm{~Hz}$ & $250 \mathrm{~Hz}$ & $500 \mathrm{~Hz}$ & $1 \mathrm{kHz}$ & $2 \mathrm{kHz}$ & $4 \mathrm{kHz}$ & & \\
\hline CI 1 & $\varnothing$ & $\varnothing$ & $\varnothing$ & $\varnothing$ & $\varnothing$ & $\varnothing$ & 75 & 102.3 \\
\hline CI 2 & $\varnothing$ & $\varnothing$ & $\varnothing$ & $\varnothing$ & $\varnothing$ & $\varnothing$ & 85 & 99.8 \\
\hline CI 3 & 70 & 70 & 65 & 85 & $\varnothing$ & $\varnothing$ & 94 & 88.2 \\
\hline CI 4 & 50 & $65(85)$ & 70 & 100 & $\varnothing$ & $\varnothing$ & 82 & 93.0 \\
\hline CI 5 & $\varnothing$ & $\varnothing$ & $\varnothing$ & $\varnothing$ & $\varnothing$ & $\varnothing$ & 94 & 117.8 \\
\hline CI 6 & $\varnothing$ & $\varnothing$ & $\varnothing$ & $\varnothing$ & $\varnothing$ & $\varnothing$ & 95 & 96.4 \\
\hline CI 7 & 100 & $\varnothing$ & $\varnothing$ & $\varnothing$ & $\varnothing$ & $\varnothing$ & 80 & 93.5 \\
\hline CI 8 & $\varnothing$ & $\varnothing$ & $\varnothing$ & $\varnothing$ & $\varnothing$ & $\varnothing$ & 80 & 98.0 \\
\hline CI 9 & $\varnothing$ & $\varnothing$ & $\varnothing$ & $\varnothing$ & $\varnothing$ & $\varnothing$ & 85 & 95.6 \\
\hline CI 10 & $\varnothing$ & 80 & 75 & 90 & $\varnothing$ & $\varnothing$ & 85 & 104.4 \\
\hline CI 11 & $\varnothing$ & $\varnothing$ & $\varnothing$ & $\varnothing$ & $\varnothing$ & $\varnothing$ & 91 & 81.1 \\
\hline CI 12 & 90 & 90 & 90 & $\varnothing$ & $\varnothing$ & $\varnothing$ & 90 & 117.84 \\
\hline CI 13 & $80(85)$ & 80 & 80 & 80 & 85 & $\varnothing$ & 67 & 78.4 \\
\hline
\end{tabular}

\section{Signal processing}

The signal processing for the experiment was similar to that of (Başkent, 2012). The speech stimuli were interrupted in two ways: with periodic silent intervals, or with the silent intervals filled with noise bursts. Interruptions were applied by modulating the sentences with a periodic, $1.5-\mathrm{Hz}$ square wave with duty cycles of either $50 \%$ or $75 \%$ of on-durations (corresponding to $333 \mathrm{~ms}$ and $500 \mathrm{~ms}$, respectively), ramped with $5 \mathrm{~ms}$ of raised cosine. Different duty cycles were used to manipulate the intelligibility of the intact portions of the sentences independently of the spectro-temporal resolution. The rate of interruption was chosen on the basis of the past study (Başkent, 2012) to produce a significant PhR effect. Duty cycles were chosen based on our previous studies (Bhargava and Başkent, 2011; Bhargava et al., Submitted), to produce sufficient intelligibility levels with interrupted speech with the both NH and CI participants. For noise conditions, a speech-shaped, steady noise was used. This noise is provided with the corpus and produced from the long-term speech spectrum of the sentences. Applying the same - but inverted - periodic square wave produced the filler noise bursts. The noise level in the interruptions was set at the signal-to-noise ratios of $-10,-5,0$ and $5 \mathrm{~dB}$ with respect to the speech portions (based on Başkent et al., 2010). Since $\mathrm{PhR}$ is a small effect with large standard deviation, and prone to individual variations even within normal-hearing listeners (Benard et al., Submitted), we took advantage of the observation that it is expected to occur over a range of conditions (Powers and Wilcox, 1977). 
As a result, we preferred to use multiple SNRs and duty cycle conditions to observe overall patterns, instead of multiple repetitions of fewer conditions.

In order to investigate the effect of spectral and temporal degradation only, in the absence of any other factor introduced by actual CIs, NH listeners were tested with noise-band vocoded speech (NHCI) in addition to non-vocoded speech (NHnorm). Fishman et al. (1997) and Friesen et al. (2001) previously showed that CI users make use of only 4 to 8 spectral channels, regardless of the number of active electrodes. Further, Friesen et al. (2001) reported that speech recognition by NH listeners with 8-channel acoustic CI simulation is comparable to that of good-performing CI users. Based on these observations and because we had selected relatively good CI users, the speech stimuli were processed with an 8-channel noise-band vocoder to produce an acoustic simulation of CIs (Başkent and Chatterjee, 2010; Başkent, 2012; Chatterjee et al., 2010; Dudley, 1939; Shannon et al., 1995). Using a bank of bandpass Butterworth filters, (order $6,36 \mathrm{~dB} /$ octave) the speech signal was filtered into 8 channels, with a total bandwidth of $150 \mathrm{~Hz}$ to $7 \mathrm{kHz}$. The matching cutoff frequencies for the analysis were determined based on Greenwood's mapping function (Greenwood, 1990) using an average cochlear length of $35 \mathrm{~mm}$ and an equal cochlear distance between filter cutoffs. Halfwave rectification and a low-pass Butterworth filter $(18 \mathrm{~dB} /$ octave, with cutoff frequency of $160 \mathrm{~Hz}$ ) were used to extract the amplitude envelope of the waveform from the analysis filters. Carrier noise bands were produced by filtering white noise with the same analysis filters. The noise carrier of each channel was then modulated using the extracted envelope for that channel. In the NHCI condition, the vocoded signal was finally produced by combining the amplitude modulated noise bands from all the channels.

In NHCI condition, the vocoding was applied to sentences after the interruptions in order to imitate the case of an actual CI user listening to interrupted stimuli more realistically.

\section{Experimental set up and procedure}

For all sessions, the participants were seated in an anechoic chamber, facing a computer monitor and a loudspeaker located at a distance of approximately 1 meter. The speech signal was processed with MATLAB, routed through the S/PDIF output of an external soundcard, Echo AudioFire 4 (Echo Digital Audio Corporation, California, USA) and presented free field through a single active Tannoy Precision 6D (digital) loudspeaker (Tannoy Ltd., UK). The speech portions of the stimuli were presented at $60 \mathrm{~dB}(\mathrm{~A})$ whereas the level of the noise was set to $55,60,65$ or $70 \mathrm{~dB}(\mathrm{~A})$ according to the noise condition.

The experiment commenced with the measurement of the VU baseline score followed by the training and the main experiment. For each participant, the $\mathrm{PhR}$ part, in the form of intelligibility task, was run before the continuity illusion task, on the same day. The main reason for this order was the limited number of sentences available in the database. The intelligibility of sentences is sensitive to learning effects, while the continuity illusion is less so. For NH listeners, the NHnorm followed by the NHCI data were collected on two different days in that order. For CI listeners, every condition was measured twice in two different sessions held on two different days. For all participants, the entire procedure lasted less than 6 hours, spread over the two sessions.

For familiarization with the procedure of the experiment, a short training with different signal-processing parameters from the main experiment $(1 \mathrm{~Hz}$ interruption rate at $40 \%$ duty cycle and $10 \mathrm{~dB}$ SNR) was provided for the intelligibility and continuity tasks. The list used for training was the same for all participants. Non-vocoded speech was used for training for the NHnorm conditions and CI users whereas vocoded speech was used for training for the NHCI conditions. During the training for the intelligibility task, participants listened to and repeated the stimulus. Feedback was provided by replaying the uninterrupted non-vocoded 
stimulus while displaying the correct text of the sentence (Benard and Başkent, 2013). In case of NHCI, the feedback was provided by replaying first uninterrupted vocoded and then uninterrupted non-vocoded stimulus while displaying the correct text of the sentence. During the training for the continuity task, each sentence was played in four formats: (i) uninterrupted without noise, (ii) uninterrupted with noise bursts, (iii) interrupted without noise, and (iv) interrupted with noise bursts in interruptions. The first two were presented as the tokens of the 'continuous' and the other two as the tokens of the 'broken' stimulus.

For the intelligibility experiment, in which the PhR benefit was measured, the participants heard the interrupted (and vocoded) sentences and verbally reported what they heard. The participants were encouraged to guess as much as they could when they were unsure. The spoken responses were recorded with a DR-100 Tascam digital voice recorder (California, USA), which were then scored offline. The participants could request the next stimulus with the help of a user interface on the monitor. The elderly CI users, who could not use the user interface, verbally cued the experimenter for presenting the next stimulus. All the words in a list were used in scoring. The percent-correct scores were calculated as the ratio of the number of correctly identified words to the number of total words in each list and averaged per list and then converted to rationalized-arcsine-unit (RAU) scores to reduce saturation effects and help restore homoscedasticity (Studebaker, 1985). There was no penalty for an incorrect or absent response. No feedback was provided during the main experiment.

For the continuity experiment, where CoI perception was measured, the participants listened to a sentence and indicated whether the sentence was continuous or broken, (if applicable). The ratio of the number of sentences judged as continuous to the total number of sentences presented was calculated and then converted to RAU scores.

Each session comprised 10 runs ( 2 duty cycles $\times 5$ SNRs). For each run, one list of 13 sentences was used. Different lists were used in the two sessions. To provide a preview of the run, every list of sentences was preceded by an introductory sentence, which was processed in the same way as the processing of the oncoming list. This introductory sentence was always the same in all the runs for all the participants and it was not included in the calculation of intelligibility. The order of the runs within each session was randomized for each participant.

Note that using the same material for the continuity task as for the intelligibility task might artificially inflate the continuity scores. However, since this would affect all the participants equally, it may not have any consequence on the comparisons between groups or conditions.

\section{E. Statistical analysis}

To assess PhR benefit and CoI perception, the scores measured with added noise were compared to the scores measured with silent gaps, using Dunnett's test for multiple comparisons. This test corrects for multiple comparisons of t-test. A significant improvement in intelligibility as a result of added noise indicated benefit from $\mathrm{PhR}$ and a significant improvement in "continuous" judgments indicated perception of CoI.

Repeated measures ANOVA (with Greenhouse-Geisser correction) was performed to observe the differences in the variances between the groups. A multiple observation correlation analysis was made to investigate if high $\mathrm{PhR}$ benefit corresponded with high CoI perception. 


\section{RESULTS}

Fig. 1 presents the average results for the experiment. The top panels show the intelligibility scores for interrupted speech with or without added filler noise, for the three modes of hearing, i.e. $\mathrm{CI}, \mathrm{NH}$ listening to normal speech (NHnorm) and $\mathrm{NH}$ listening to acoustic simulation of CI speech (NHCI). Similarly, the bottom panels show scores for the continuity judgment task. The VU baseline scores, shown by the black lines in the top panels, are comparable for $\mathrm{CI}$ and $\mathrm{NHCI}$, confirming that the selection of participants was appropriate.
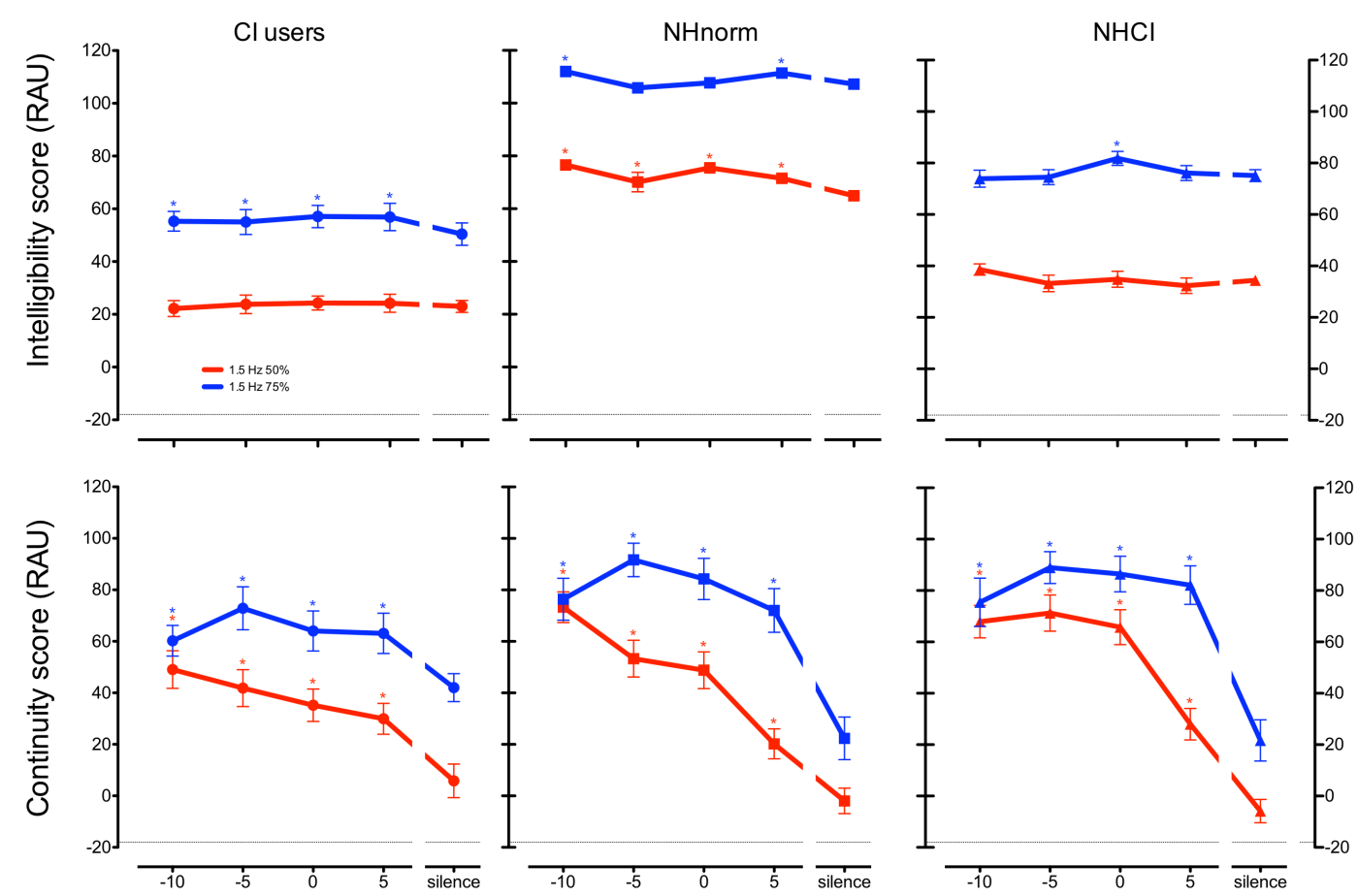

SNR in interruption

Figure 1: The mean intelligibility scores (top panels) and continuity scores (bottom panels) plotted in RAU, and shown for each participant group as a function of speech to noise ratio (SNR). Dotted red and solid blue lines show the results for the $50 \%$ and $75 \%$ duty cycle, respectively. SNR conditions with a significant increase in performance compared to the silence condition, as determined by a Dunnett's test, are marked with an asterisk. The error bars denote one standard error. The black lines in top panels for $\mathrm{CI}$ and NHCI represent the VU baseline scores measured with uninterrupted stimuli. These were not measured for NHnorm because NH listeners were expected to entirely understand normal speech in the lack of acoustic CI simulation.

\section{A. Phonemic restoration benefit}

In Fig. 1, top panels, the PhR benefit appears when speech intelligibility in the noise conditions (data points to the left) is higher than in the silent gap condition (the rightmost data point in each panel).

We first inspect the intelligibility data with the $50 \%$ duty cycle (Fig. 1, top panels, dotted red lines). $\mathrm{NH}$ listeners presented with normal non-vocoded speech (NHnorm) representing the control group (Fig. 1, top middle panel) showed significant PhR benefit (8.6 RAU, averaged across all SNRs) at all SNR conditions [for all comparisons, $p<0.05$ ]. NH listeners presented with acoustic CI-simulation (NHCI condition) were used in this study to see the effect of only 
spectral and temporal degradations on $\mathrm{PhR}$ (Fig. 1, top right panel). No significant $\mathrm{PhR}$ benefit (0.3 RAU on average) was observed for any of the SNR conditions [for all comparisons, $p \geq 0.059$ ]. Thus, at the 50\% duty cycle, NH listeners showed a significant $\mathrm{PhR}$ benefit with non-vocoded speech, but not with vocoded speech, a pattern that has been previously reported for similar test conditions (Başkent, 2012, 2010; Başkent et al., 2010). Importantly, similar to acoustic CI simulations, CI users (Fig. 1, top left panel) also showed no significant PhR benefit ( $0.6 \mathrm{RAU}$ on average) for the $50 \%$ duty cycle [for all comparisons, $p \geq 0.62]$.

The intelligibility data for the $75 \%$ duty cycle has a different pattern (Fig.1, top panels, blue lines). An increased duty cycle was used to test if making speech segments longer to increase the amount of bottom-up speech cues would help with top-down restoration. With the $75 \%$ duty cycle, the intelligibility of interrupted speech with gaps by NH listeners for NHnorm mode of hearing was very close to ceiling (Fig. 1, top middle panel), leaving little room for more improvement due to $\mathrm{PhR}$. Indeed, the PhR benefit was significant only at two SNR conditions [4.5 RAU average at the two SNRs $-10 \mathrm{~dB}$ and $5 \mathrm{~dB}, p<0.05$ ] and not significant for the others [-0.4 RAU on average over -5 and $0 \mathrm{~dB}$ SNR, $p \geq 0.66]$. NHCI mode of hearing (Fig. 1, top right panel) showed no significant PhR benefit except for one SNR condition [6.7 RAU at $0 \mathrm{~dB}$ SNR, $p<0.05$; 0.3 RAU on average over all other SNR conditions, $p \geq 0.92]$ at this duty cycle. Unlike NHCI, the users of CI were able to show significant PhR benefit for all SNRs for this duty cycle. Since the NHCI scores were not close to ceiling, the absence of PhR benefit for this mode of hearing could not have been due to a saturation effect like it was in the case of NHnorm. The absence of PhR benefit also could not have been caused by the scores in the silent condition being too low, as at the same interruption condition, CI users (Fig. 1, top left panel) scored even lower (75.1 RAU for NHCI vs. 50.4 RAU for CI) and yet showed significant PhR benefit [5.6 RAU on average, for all comparisons, $p<0.05$ ].

Thus, at the lower duty cycle, CI users could not benefit from the addition of noise into the silent intervals, but with the higher duty cycle, they showed this benefit. Perhaps for the CI users, higher duty cycle provided more speech cues for activation of relevant knowledge in order to repair the interrupted speech. Unlike the CI users, the $\mathrm{NH}$ listeners presented with vocoded speech could not attain a significant PhR benefit consistent across all SNRs (except at $0 \mathrm{~dB}$ SNR) even with more speech cues available with the higher duty cycle.

In order to see if $\mathrm{PhR}$ benefit remained absent at the $50 \%$ duty cycle for all CI users, we investigated the individual data: 6 participants showed some PhR benefit, 6 showed a deficit and 1 showed no visible benefit or deficit (see Fig. 2). We explored, within the CI group, whether it was the better performing CI users who obtained more PhR benefit. A correlation analysis between the VU baseline scores of uninterrupted stimuli and the PhR benefits at the two duty cycles was conducted. Fig. 2 presents the linear regression between the VU baseline scores and PhR benefit at the 50\% duty cycle (left panel) and the $75 \%$ duty cycle (right panel) for CI users. A significant and strong correlation was found between the VU baseline scores and $\mathrm{PhR}$ benefit for the $50 \%$ duty cycle $[\mathrm{r}=0.71, p<0.05]$ but not for the $75 \%$ duty cycle $[\mathrm{r}$ $=0.13, p=0.67]$. In fact, the data points in Fig. 2 (left panel) shows that for the 50\% duty cycle, more than half of the CI users, i.e., the better performing ones according to the VU baseline, showed some amount of PhR benefit. However, because the other half showed a negative effect of adding noise, the overall average PhR benefit came out as non-significant. For the $75 \%$ duty cycle, most of the CI users were able to attain PhR benefit notwithstanding their VU baseline scores, which resulted in significant overall PhR benefit, and no significant correlation was observed. 


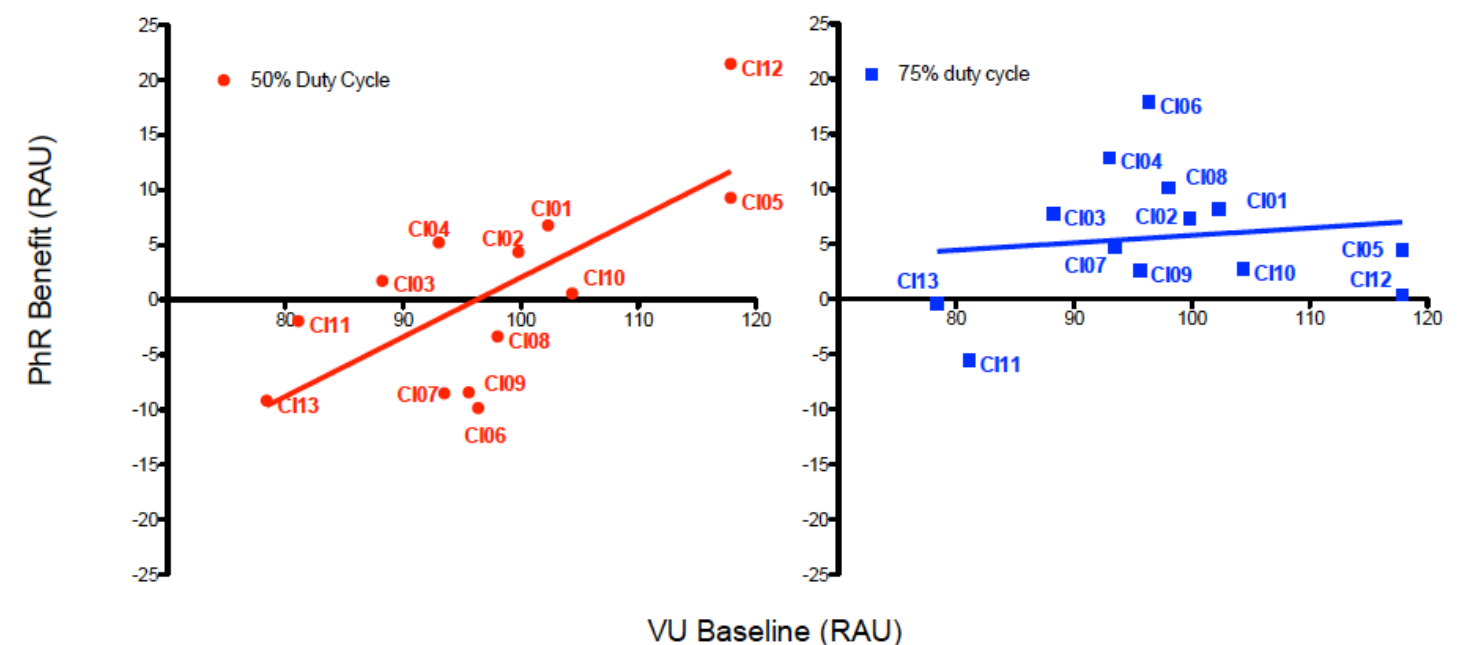

Figure 2: PhR benefit shown as a function of the VU baseline scores of sentence recognition and labeled for individual CI users. Red (left panel) and blue (right panel) symbols represent the 50\% and $75 \%$ duty cycle, respectively.

We did a number of additional analyses to see what demographic factors from CI users may have affected the speech intelligibility performance, as well as $\mathrm{PhR}$ benefit. The duration of $\mathrm{CI}$ use and the VU baseline scores were found to correlate moderately but significantly $[\mathrm{r}=$ $0.58, p=0.039$ ]. For the $50 \%$ duty cycle, the correlation between the duration of CI use and $\mathrm{PhR}$ benefit was found to correlate moderately and significantly $[\mathrm{r}=0.63, p=0.021]$, but not for the $75 \%$ duty cycle $[r=0.12, p>0.05$. Hence, at the longer duty cycle, most CI users seem to attain restoration benefits, however, at the shorter duty cycle, this benefit seems to be limited to some individual CI users, depending on how well they perform in general. Hearing aid use did not show an effect on PhR benefit (a repeated measures ANOVA with between subject factor of 'hearing aid use'; $\mathrm{F}_{1,11}=0.21, p=0.66$ ).

Combined with the results from Dunnett's test showing that CI users are capable of obtaining $\mathrm{PhR}$ benefit when more speech features can be transmitted (at the $75 \%$ duty cycle), the correlation analysis also shows that some CI users are even able to obtain $\mathrm{PhR}$ benefit with sparse speech cues and in the same conditions as NH listeners, depending on their experience with their device and their VU baseline performance.

\section{B. Continuity illusion}

The second mechanism potentially involved in the top-down restoration was CoI. The lower panels of Fig. 1 show the RAU scores for perceived continuity for interrupted speech with silent intervals, as well as with the filler noise, for both duty cycles. The figure shows that, as expected, the sentences interrupted at the $75 \%$ duty cycle were in general perceived as more continuous than with the $50 \%$ duty cycle, perhaps due to the interruptions being shorter. With silent intervals, the $50 \%$ duty cycle condition was almost never judged as continuous (around 0 RAU continuity score for all groups). However, with the $75 \%$ duty cycle there was a greater continuity perception with continuity judgment closer to the chance level of 50 RAU for CI users (42.0 RAU). For the other two modes of hearing, the continuity score (around $22 \mathrm{RAU}$ ) was higher than no-continuity (-18 RAU) but lower than chance level (50 RAU). As speculated before, this might be due to the $75 \%$ duty cycle gaps being short in duration.

CoI perception was measured as the increase in perceived continuity due to the addition of noise, i.e., the increase in reported continuity at SNR conditions compared to the silent conditions. The results reveal that for all groups and both duty cycles, the continuity at all 
SNRs was greater than that of sentences with silent intervals, revealing a significant CoI perception [for all comparisons, $p<0.05$ ]. Thus, the addition of filler noise to the silent intervals made the interrupted speech sound more continuous for all modes of hearing at both duty cycles.

Despite the existence of a significant continuity illusion for all the groups, there were some differences observed between the CI group and the others. A repeated measures ANOVA with CoI perception data from NHnorm and NHCI modes of hearing, with duty cycle, SNR and simulation as within-subject factors revealed no significant effect of simulation (Table III). Thus, adding the acoustic CI simulation to the stimuli did not change CoI, as the results between NHnorm and NHCI groups were very similar with or without added noise for both duty cycles. CI users, on the other hand, showed a slightly different trend. They heard sentences with gaps at the $75 \%$ duty cycle more often (and erroneously) as continuous than both NHnorm and NHCI listeners (42 for CI vs. 22 RAU for NHnorm and NHCI), and they also reported weaker continuity with the added noise than the other two groups. Repeated measures ANOVAs comparing CoI perception data from CI and NHnorm modes of hearing (Table IV), and CI and NHCI modes of hearing (Table V), with duty cycle and SNR as within-subject factors and mode of hearing as between-subject factor revealed a significant effect of mode of hearing. Hence, for CoI, while the degradations of Acoustic CI simulation did not cause a change in results compared to $\mathrm{NH}$, the use of an actual CI device did, which could not be explained on the basis of only the impoverished spectral resolution induced by CI

processing.

TABLE III. Repeated measures three-way ANOVA on CoI scores of NH listeners presented with normal speech (NHnorm) and presented with acoustically CI-simulated speech (NHCI). SNR conditions, duty cycle, and acoustic CI simulation were within-subject factors.

\begin{tabular}{|l|l|r|}
\hline Source & $\mathrm{F}$ value & Significance $(\mathrm{p})$ \\
\hline SNR & $\mathrm{F}(3,39)=28.38$ & $<0.001$ \\
\hline Duty Cycle & $\mathrm{F}(1,13)=0.2$ & 0.66 \\
\hline CI simulation & $\mathrm{F}(1,13)=1.09$ & 0.31 \\
\hline CI sim $\times$ Duty cycle & $\mathrm{F}(1,13)=2.74$ & 0.12 \\
\hline CI sim $\times$ SNR & $\mathrm{F}(3,39)=1.16$ & 0.34 \\
\hline Duty cycle $\times$ SNR & $\mathrm{F}(3,39)=13.18$ & $<0.001$ \\
\hline CI sim $\times$ Duty cycle $\times$ SNR & $\mathrm{F}(3,39)=2.72$ & 0.57 \\
\hline
\end{tabular}

TABLE IV. Repeated measures three-way ANOVA on CoI scores of NH listeners presented with normal speech (NHnorm) and CI users. SNR conditions and duty cycle were within-subject factors and mode of hearing between-subject factor.

\begin{tabular}{|l|l|r|}
\hline Source & $\mathrm{F}$ value & Significance $(\mathrm{p})$ \\
\hline SNR & $\mathrm{F}(3,75)=18.74$ & $<0.001$ \\
\hline Duty Cycle & $\mathrm{F}(1,25)=0.05$ & 0.83 \\
\hline Mode of hearing & $\mathrm{F}(1,25)=7.12$ & $<0.05$ \\
\hline Mode of hearing $\times$ Duty cycle & $\mathrm{F}(1,25)=3.08$ & 0.092 \\
\hline Mode of hearing $\times$ SNR & $\mathrm{F}(3,75)=5.05$ & $<0.05$ \\
\hline Duty cycle $\times$ SNR & $\mathrm{F}(3,75)=14.55$ & $<0.001$ \\
\hline Mode of hearing $\times$ Duty cycle $\times$ SNR & $\mathrm{F}(3,75)=1.86$ & 0.14 \\
\hline
\end{tabular}


TABLE V. Repeated measures three-way ANOVA on CoI scores of NH listeners presented with acoustically CI-simulated speech (NHCI) and CI users. SNR conditions and duty cycle were within-subject factors and mode of hearing between-subject factor.

\begin{tabular}{|l|l|r|}
\hline Source & F value & Significance $(\mathrm{p})$ \\
\hline SNR & $\mathrm{F}(3,75)=8.04$ & $<0.001$ \\
\hline Duty Cycle & $\mathrm{F}(1,25)=1.67$ & 0.21 \\
\hline Mode of hearing & $\mathrm{F}(1,25)=11$ & $<0.05$ \\
\hline Mode of hearing $\times$ Duty cycle & $\mathrm{F}(1,25)=0.61$ & 0.44 \\
\hline Mode of hearing $\times$ SNR & $\mathrm{F}(3,75)=2.15$ & 0.10 \\
\hline Duty cycle $\times$ SNR & $\mathrm{F}(2.2,54.93)=9.31$ & $<0.001$ \\
\hline Mode of hearing $\times$ Duty cycle $\times$ SNR & $\mathrm{F}(3,75)=2.72$ & 0.051 \\
\hline
\end{tabular}

\section{Phonemic restoration and continuity illusion correlation}

The previous two sections described the findings on $\mathrm{PhR}$ benefit and CoI perception separately. The differences observed in the patterns between the PhR benefit and CoI perception questioned the assumption of how much their underlying mechanisms overlapped. To investigate this, a multiple observation correlation analysis was conducted between the two. A multiple observation correlation coefficient was calculated for within and between subjects (J M. Bland and Altman, 1995; J. M. Bland and Altman, 1995). The within-subject correlation analysis was performed to see whether or not for each mode of hearing, for individual participants, an increase in $\mathrm{PhR}$ benefit with respect to duty cycle and SNR corresponds to an increase in CoI perception. A weak, but significant, negative correlation was found between the PhR benefit and $\mathrm{CoI}$ for the CI users $(\mathrm{r}=-0.24, p=0.02)$. No significant correlation was found for the NHnorm $(\mathrm{r}=0.16, p=0.11)$ and NHCI conditions $(\mathrm{r}$ $=0.034, p=0.73)$. The between-subject correlation analysis was performed to see if individuals with overall high $\mathrm{CoI}$ also tended to have overall high $\mathrm{PhR}$ benefit. The betweensubject correlation coefficients for all the three modes of hearing were found to be nonsignificant (CI users, $\mathrm{r}=0.18$; NHnorm, $\mathrm{r}=-0.04$; NHCI, $\mathrm{r}=0.24$; for all, $p \geq 0.39$ ). Thus, for CI users, an increase in CoI was actually accompanied by a weak decrease in the PhR benefit, but no such relationship could be significantly established for other modes of hearing. With the $75 \%$ duty cycle, the CI users obtained greater PhR benefit, but the silent gaps at this duty cycle sounded more continuous to them, which brought down the CoI. This may explain the weak negative correlation for CI users. Thus, overall results showed no clear relationship between CoI perception and PhR benefit, and the only significant correlation goes in the opposite direction to what was predicted from the literature.

\section{DISCUSSION}

The hypothesis of the present study was that the perceptual restoration of masked or interrupted speech from the top-down processes might be restricted in CI users, due to the reduced quality of bottom-up speech cues. These could be degraded or changed due to several factors related to CIs, such as loss of spectral resolution or temporal fine structure in speech signals transmitted, but also front-end processing, health of spiral ganglion, electrode placement, and frequency-place mismatch. This hypothesis was explored measuring $\mathrm{PhR}$ and $\mathrm{CoI}$ in $\mathrm{CI}$ users and comparing the results to those of $\mathrm{NH}$ listeners. $\mathrm{NH}$ listeners were also presented with 8-channel noiseband vocoded speech in order to see the effect of degraded speech signals due to CI signal processing alone, without involving other CI-related factors. 


\section{A. Phonemic restoration}

The data for CI users suggest that for several conditions, the addition of noise to the silent intervals does result in a significant increase in intelligibility. At the $75 \%$ duty cycle significant average $\mathrm{PhR}$ benefit was found and, although at the $50 \%$ duty cycle no significant average PhR benefit was found, a few participants with high VU baseline performance and long duration of CI use did show PhR benefit. Thus, when fewer speech features were available, the VU baseline, a measure of general performance level of a CI user, could predict whether a CI user obtained PhR benefit or not. However, when more speech features were available, most $\mathrm{CI}$ participants obtained $\mathrm{PhR}$ benefit, and the VU baseline was not predictive anymore. Perhaps the CI users with higher VU baseline, also have better access to more speech cues in general, or they are able to hear more subtle speech cues, or perhaps they make better use of whatever speech cues that they can access. All of these could also help with obtaining a better PhR benefit in more challenging conditions of shorter duty cycles.

These observations with CI users were somewhat different than that of NHnorm, where significant $\mathrm{PhR}$ effect was observed at the $50 \%$ duty cycle, in line with previous studies (Bashford and Warren, 1979; Başkent et al., 2009; Benard and Başkent, 2013; Powers and Wilcox, 1977; Verschuure and Brocaar, 1983), and at selected SNRs (likely due to ceiling effect) at the $75 \%$ duty cycle. Hence, the pattern and extent of PhR benefit differed in CI users as compared with NH listeners, but it was not completely diminished as would be expected based on previous simulation studies (e.g., Başkent, 2012).

In CI users, that a general $\mathrm{PhR}$ benefit was observed at the higher duty cycle may seem to indicate that the extent of $\mathrm{PhR}$ benefit depends on the intelligibility of interrupted speech, as this was also higher at the higher duty cycle. However, Başkent (2010) showed that the extent of $\mathrm{PhR}$ does not depend on the level of intelligibility of speech with silent interruptions, which was also indirectly supported by data by Verschuure and Brocaar (1983). Hence, the intelligibility of interrupted speech seems to be an insufficient explanation for the pattern observed in results with CI users.

There is, however, a more plausible explanation for why increasing the duty cycle may have helped the CI users benefit more from the PhR, namely, the kind of speech features that are transmitted in CIs and how they are effectively utilized by the users. The speech transmitted from the CI to the brain lacks fine spectral and temporal structures, and only retains the temporal envelopes from a number of spectral bands (Loizou, 1998; Shannon et al., 1995). Therefore, CI users heavily rely on temporal cues (Fu et al., 2004; Nie et al., 2006; Rosen, 1992; Tasell et al., 1992). These temporal cues are crucial in providing information about segmental (e.g. voicing and manner cues) and suprasegmental (e.g. syllabification, word onset and offset times, speaking rate and prosody) speech features on which intelligibility of speech rests (Assmann and Summerfield, 2004; Fogerty and Humes, 2012; Shannon et al., 1995). The studies done with vowel-only and consonant-only speech have also shown that speech envelope provides acoustic cues that help sentence comprehension by facilitating top-down processing (Fogerty and Humes, 2012; Fogerty and Kewley-Port, 2009). In the absence of fine structure, all envelope cues, and the speech features they code, become essential for CI users to understand speech. These cues would be better delivered and transmitted by longer segments of speech, thus facilitating better lexical activation. Hence, it is more likely that with higher duty cycle, CI listeners received the kind of speech features they can use for restoration.

Stilp et al. (2013) alternatively argued that it is not specifically the envelope per se that is bearing information, but spectral changes as captured by the cochlea-scaled entropy which is indirectly related to the envelope. Cochlea-scaled entropy is a measure of (un)predictability of spectral change in signals in slices of fixed duration based on preceding slices. It encodes information as change over time. More generally, Stilp and Kluender (2010) have shown that 
sentence portions with higher cochlear-entropy are more important for speech intelligibility than portions with low cochlear-entropy. Thus, not all portions of speech signal are equally important for intelligibility. Thus, PhR may not depend only on the sheer amount of speech features but also on the kind of speech features available to the listener and their relative value for intelligibility.

However, these considerations do not explain the results of the acoustic CI-simulation condition (NHCI). For this mode of hearing, like for the CI users, no significant $\mathrm{PhR}$ benefit was observed at the $50 \%$ duty cycle, which is also consistent with the findings of Başkent (2012). But unlike for the CI users, no significant PhR benefit was observed at the $75 \%$ duty cycle either (except at $0 \mathrm{~dB}$ SNR), even though overall intelligibility was much higher than at the $50 \%$ duty cycle. This again confirms that the overall intelligibility does not seem to be the primary determinant of PhR benefit (Başkent, 2012; Verschuure and Brocaar, 1983).

Further theoretical considerations about the underlying mechanisms of $\mathrm{PhR}$ may help explain these results. Srinivasan and Wang (2005) proposed that distinguishing speech from noise, in order to identify speech segments, is necessary for PhR to work. These identified speech segments provide the linguistic context that constrains the lexical activation of schemas (Huggins, 1964; Srinivasan and Wang, 2005). Silent interruptions in speech could be introducing spurious speech cues, e.g., initiating word segmentation, imitating stop consonants or glottal closures, etc. (Huggins, 1964; Repp et al., 1978). In this case, the listeners do not have the choice but to take into account these wrong speech cues, which could lead to favoring wrong lexical possibilities. Intervening noise may not only give rise to CoI by directly hiding the silent gaps, the evidence of discontinuity, but may also prevent the formation of these spurious cues. The absence of these spurious cues, in turn, leaves more freedom to the top-down mechanisms to produce the appropriate schemas that can fit the evidence extracted from the presented speech segment. Since, in presence of noise, the selected solution is based exclusively on the content of the speech segment rather than also on spurious cues, this solution has a higher possibility to be accurate, thus improving intelligibility (Srinivasan and Wang, 2005). According to this explanation, it would be important for the auditory system to be able to distinguish between noise and speech. If speech sounds noise-like, the auditory system may confuse the noise with speech cues, thus again introducing spurious cues, hence not producing an improvement in intelligibility. This conclusion is in agreement with the findings of other studies. For example, Clarke et al. (2013) reported a change in the PhR when they used different parameters of vocoding. Similarly, using zebra-speech (a purely sequential mixture of speech and interfering signal), Gaudrain and Carlyon (2013) found that when there was no qualitative difference between the target (noise-band vocoded speech) and the interfering sounds (speech-shaped noise) there was more informational masking, which resulted in a loss of intelligibility. Since CI processing does not use noise carriers but pulse trains, CI users likely had better discrimination between noise and speech and indeed showed a significant $\mathrm{PhR}$ benefit. However, if the electrical stimulation creates a percept of speech and noise that are more similar to each other qualitatively, there is a possibility that this can also contribute to reduced restoration in actual CI users as well.

Note that there were two factors that differed between $\mathrm{CI}$ and $\mathrm{NH}$ groups that may have affected the results. One is that the average age of CI group was considerably higher than the average age of $\mathrm{NH}$ group, and due to aging effects on cognitive processes, one can suspect that age could also play a role in $\mathrm{PhR}$ benefit. A negative effect of age was previously shown on perception of interrupted speech with silent gaps (Gordon-Salant and Fitzgibbons, 1993; Kidd and Humes, 2012; Saija et al., 2013). However, despite this, Saija et al. (2013) recently observed that older individuals showed PhR benefit to a degree similar to that of younger individuals. Thus, the ability of top-down restoration seems to remain robust for older individuals, and therefore, we did not expect age to play a major role in the findings of the present study. Another difference between CI and NH groups is that CI users have more 
experience in listening to degraded speech than $\mathrm{NH}$ listening to noise-vocoded speech (NHCI). In relatively short-term but intense training studies, Benard and Başkent (2013; submitted) observed a significant overall learning of interrupted speech both with or without filler noise, and with or without vocoding. However, $\mathrm{PhR}$ beneft did not appear or disappear as a result of training; despite the overall performance increase, the existence (or lack of, depending on the experimental condition) of $\mathrm{PhR}$ remained the same at the end of training as it was at the beginning. Longer-term exposure may have had some stronger learning effects, but these would not be possible to address in well-controlled lab experiments.

\section{B. Continuity illusion}

For all modes of hearing, the continuity in the silent condition was judged higher with the $75 \%$ duty cycle than the $50 \%$ duty cycle even though there was no noise hiding the silent interruptions (Fig.1, lower panels). This means that with the $75 \%$ duty cycle, on some occasions, the listeners were not able to register the silent intervals. One possibility is that this is caused by the shorter duration of gaps. Or, more interestingly, and consistent with the discussion in the previous section, the participants were misinterpreting the silent intervals as natural parts of the speech. Once the filler noise was added to the silent intervals, a significant increase in CoI was observed for all modes of hearing, for all SNRs and for both duty cycles. Hence, adding the noise indeed strengthened perceived continuity, as was expected.

One of the expectations associated with acoustic CI-simulation (NHCI) and CI-processed (CI) speech was that the degradation of the signal quality due to the loss of spectral resolution and temporal fine structure might enforce stronger fusion of the speech and noise segments. This may then result in increased CoI perception. Contrary to this expectation, NH listeners obtained almost equal and significant $\mathrm{CoI}$ with (NHCI) and without (NHnorm) the acoustic simulation of CI for both duty cycles. CI users also obtained significant CoI perception. But, contrary to expectation, at the $75 \%$ duty cycle, their CoI perception was weaker than that of $\mathrm{NH}$ listeners, due to (erroneously) judging the silent interruptions as continuous almost up to the chance level. CoI perception at the $75 \%$ duty cycle was also weaker than at the $50 \%$ duty cycle.

For CoI, NHnorm and NHCI data were similar to each other, but different from the data from CI users. This indicates that reduction in signal quality per se is not sufficient to affect CoI, while it mattered for intelligibility and PhR benefit. In actual CI users, there may be many more factors e.g. front-end processing of the CIs, as well as the health of spiral ganglion, the potential current spread, frequency-place mismatch in mapping, that may be affecting $\mathrm{CoI}$ in $\mathrm{CI}$ users in addition to the signal degradations. An example of potential effects of front-end processing on CoI was shown for simulated dynamic range compression by Başkent et al. (2009). Additionally, since the NH participants were young and CI participants were older in age, CoI results may have been affected by age. Saija et al. (2013) had only shown no age effect on PhR benefit, but they did not study this for CoI. EEG studies have provided evidence that aging affects temporal integration in elderly listeners (Bertoli et al., 2002; Horváth and Burgyán, 2011). Behavioral studies have reported larger gap thresholds and gap duration difference limens for older listeners (Lister and Tarver, 2004; Lister et al., 2002; Pichora-Fuller et al., 2006; Strouse et al., 1998). As these studies suggest that temporal integration and temporal processing may be sensitive to aging effects, one can conceive that perceiving gaps in interrupted speech could potentially be affected by age.

All combined, CI users seem to have more difficulty judging if a speech signal is discontinuous or continuous. This does not seem to have a considerable effect on $\mathrm{PhR}$, as at the $75 \%$ duty cycle they could benefit from it, despite the weaker CoI observed at this duty cycle. However, as continuity may be an important step of perceptual grouping and 
organization in general (Best et al., 2008; Nelson and Jin, 2004; Shinn-Cunningham et al., 2013; Shinn-Cunningham and Wang, 2008), these results may also be hinting that CI users may have difficulties in benefiting from perceptual organization for better speech intelligibility in complex listening environments.

\section{Continuity illusion and phonemic restoration}

One hypothesis that associates $\mathrm{PhR}$ with $\mathrm{CoI}$ is that the addition of noise to the silent intervals gives rise to CoI, which may help in sequentially grouping parts of speech across noise bursts (Warren, 1984). As mentioned above, this may help with perceptual grouping, which in turn may help improve intelligibility (Nelson and Jin, 2004). According to this hypothesis, adding noise to silent intervals would lead to CoI perception and hence to PhR benefit (Bashford et al., 1992; Carlyon et al., 2004, 2002; Heinrich et al., 2008; Verschuure and Brocaar, 1983). While this may be true in some situations, other studies mentioned that the neural mechanisms of the two phenomena may not be hierarchical, but instead, working in parallel with some overlap (Chatterjee et al., 2010; Shahin et al., 2009). Our data give more support to the latter. We found that CI users showed significant CoI perception at both duty cycles and yet showed significant PhR benefit at only one duty cycle, incidentally, at the one with lesser CoI perception. This was also evident from the weak but significant negative correlation between PhR benefit and CoI perception for CI users. Similarly, for NH listeners, the CoI with acoustically simulated speech (NHCI) was as strong as with non-vocoded speech (NHnorm), yet PhR benefit with NHCI was not significant at most conditions as opposed to NHnorm. Correlation analyses were also not significant.

These results support the idea that continuity and repair are partially dissociable mechanisms, possibly also situated in separate cortical regions, but which communicate with each other when repair is required (Shahin et al., 2009). One can conceive a scenario where the filler noise is not perceptually similar enough to speech to entirely disguise the silent gap to produce a very strong CoI. It might instead introduce sufficient amount of ambiguity to activate the appropriate lexical possibilities once combined with the speech features of the remaining segments. Hence, our observations are more in line with the view that while the two mechanisms seem to be related (e.g. Başkent et al., 2009; Shahin et al., 2009), they might not be entirely overlapping, nor ordered in a hierarchical manner (Shahin et al., 2009).

\section{Conclusions}

In summary, the present study shows that, like NH listeners, CI users can employ top-down repair mechanisms to perform restoration of degraded speech, provided they can extract the appropriate speech features from the transmitted bottom-up speech cues. In a real-life noisy situation, the bottom-up speech cues may be further degraded than what was tested here in controlled laboratory conditions, disrupting the trigger for top-down repair mechanisms. We additionally observed a great variation in CI users' ability to understand interrupted speech and to benefit from $\mathrm{PhR}$, which seemed to be also relevant to speech recognition skills in quiet. Further, the performance of $\mathrm{CI}$ users on $\mathrm{PhR}$ and $\mathrm{CoI}$ both differed from that of $\mathrm{NH}$ listeners. These observations may partially explain why CI users have difficulty in understanding speech in noisy situations. Such interactions between top-down mechanisms and bottom-up speech cues should, therefore, perhaps be taken into account to improve future implant devices, for example, by using better tests during new device algorithm development, or by using better rehabilitation programs for CI users. 


\section{ACKNOWLEDGEMENTS}

The study was supported by a VIDI grant from the Netherlands Organization for Scientific Research, NWO, and Netherlands Organization for Health Research and Development, ZonMw (grant no. 016.096.397). Further support came from a Rosalind Franklin Fellowship from University of Groningen, University Medical Center Groningen, and funds from Heinsius Houbolt Foundation. We thank our participants for their cooperation, and Ir. Bert Maat, Floor Burgerhof, Esmée Van Der Veen and Marije Sleurink for their valuable assistance. The study is part of the research program of the Otorhinolaryngology Department of University Medical Center Groningen: Healthy Aging and Communication.

\section{REFERENCES}

Assmann, P., Summerfield, Q., 2004. The Perception of Speech Under Adverse Conditions. In: Speech Processing in the Auditory System, Springer Handbook of Auditory Research. Springer New York, pp. 231-308.

Bashford, J.A., Riener, K.R., Warren, R.M., 1992. Increasing the intelligibility of speech through multiple phonemic restorations. Percept. Psychophys. 51, 211-217.

Bashford, J.A.J., Warren, R.M., 1979. Perceptual synthesis of deleted phonemes. J. Acoust. Soc. Am. 65, S112$\mathrm{S} 112$.

Başkent, D., 2010. Phonemic restoration in sensorineural hearing loss does not depend on baseline speech perception scores. J. Acoust. Soc. Am. 128, EL169-EL174.

Başkent, D., 2012. Effect of speech degradation on top-down repair: phonemic restoration with simulations of cochlear implants and combined electric-acoustic stimulation. J. Assoc. Res. Otolaryngol. 13, 683-692.

Başkent, D., Chatterjee, M., 2010. Recognition of temporally interrupted and spectrally degraded sentences with additional unprocessed low-frequency speech. Hear. Res. 270, 127-133.

Başkent, D., Eiler, C., Edwards, B., 2009. Effects of envelope discontinuities on perceptual restoration of amplitude-compressed speech. J. Acoust. Soc. Am. 125, 3995-4005.

Başkent, D., Eiler, C.L., Edwards, B., 2010. Phonemic restoration by hearing-impaired listeners with mild to moderate sensorineural hearing loss. Hear. Res. 260, 54-62.

Başkent, D., Shannon, R.V., 2005. Interactions between cochlear implant electrode insertion depth and frequencyplace mapping. The Journal of the Acoustical Society of America 117, 1405-1416.

Başkent, D., Shannon, R.V., 2006. Frequency transposition around dead regions simulated with a noiseband vocoder. J. Acoust. Soc. Am. 119, 1156-1163.

Benard, M.R., Başkent, D., Submitted. Perceptual learning of temporally interrupted and spectrally degraded speech.

Benard, M.R., Başkent, D., 2013. Perceptual Learning of Interrupted Speech. PLoS ONE 8, e58149.

Benard, M.R., Mensink, J.S., Başkent, D., Submitted. Individual differences in top-down restoration of interrupted speech: Links to linguistic and cognitive abilities. JASA-EL.

Bertoli, S., Smurzynski, J., Probst, R., 2002. Temporal resolution in young and elderly subjects as measured by mismatch negativity and a psychoacoustic gap detection task. Clinical Neurophysiology 113, 396-406.

Best, V., Ozmeral, E.J., Kopčo, N., Shinn-Cunningham, B.G., 2008. Object continuity enhances selective auditory attention. PNAS 105, 13174-13178.

Bhargava, P., Başkent, D., 2011. Phonemic Restoration and Continuity Illusion with Cochlear Implants. Presented at the Conference on Implantable Auditory Prostheses, Asilomar Conference Grounds Pacific Grove, California.

Bhargava, P., Gaudrain, E., Başkent, D., Submitted. The intelligibility of interrupted speech: Cochlear implant users and normal hearing listeners.

Bland, J M., Altman, D.G., 1995. Statistics notes: Calculating correlation coefficients with repeated observations: Part 2--correlation between subjects. Brit. Med. J. 310, 633-633.

Bland, J. M., Altman, D.G., 1995. Calculating correlation coefficients with repeated observations: Part 1-Correlation within subjects. Brit. Med. J. 310, 446-446.

Bosman, A.J., 1989. Speech perception by the hearing impaired (Doctoral thesis). Rijksuniversiteit Utrecht.

Bregman, A.S., 1990. Auditory Scene Analysis: The Perceptual Organization of Sound. The MIT Press.

Carlyon, R.P., Deeks, J., Norris, D., Butterfield, S., 2002. The continuity illusion and vowel identification. Acta Acust. united Ac. 88, 408-415.

Carlyon, R.P., Micheyl, C., Deeks, J.M., Moore, B.C.J., 2004. Auditory processing of real and illusory changes in frequency modulation (FM) phase. The Journal of the Acoustical Society of America 116, 3629-3639.

Chatterjee, M., Peredo, F., Nelson, D., Başkent, D., 2010. Recognition of interrupted sentences under conditions of spectral degradation. J. Acoust. Soc. Am. 127, EL37-41.

Clarke, J., Gaudrain, E., Başkent, D., 2013. Top-down repair of speech: adding pitch to spectrally degraded speech. Presented at the Conference on Implantable Auditory Prostheses, Lake Tahoe, California. 
Dudley, H., 1939. Remaking Speech. The Journal of the Acoustical Society of America 11, 169-177.

Fishman, K.E., Shannon, R.V., Slattery, W.H., 1997. Speech recognition as a function of the number of electrodes used in the SPEAK cochlear implant speech processor. J. Speech Lang. Hear. Res. 40, 1201-1215.

Fogerty, D., Humes, L.E., 2012. The role of vowel and consonant fundamental frequency, envelope, and temporal fine structure cues to the intelligibility of words and sentences. The Journal of the Acoustical Society of America 131, 1490-1501.

Fogerty, D., Kewley-Port, D., 2009. Perceptual contributions of the consonant-vowel boundary to sentence intelligibility. J Acoust Soc Am 126, 847-857.

Friesen, L.M., Shannon, R.V., Başkent, D., Wang, X., 2001. Speech recognition in noise as a function of the number of spectral channels: comparison of acoustic hearing and cochlear implants. J. Acoust. Soc. Am. 110, $1150-1163$.

Fu, Q.-J., Chinchilla, S., Galvin, J.J., 2004. The Role of Spectral and Temporal Cues in Voice Gender Discrimination by Normal-Hearing Listeners and Cochlear Implant Users. J Assoc Res Otolaryngol 5, 253260.

Fu, Q.J., Shannon, R.V., Wang, X., 1998. Effects of noise and spectral resolution on vowel and consonant recognition: acoustic and electric hearing. J. Acoust. Soc. Am. 104, 3586-3596.

Gaudrain, E., Carlyon, R.P., 2013. Using Zebra-speech to study sequential and simultaneous speech segregation in a cochlear-implant simulation. J. Acoust. Soc. Am. 133, 502-518.

Gordon-Salant, S., Fitzgibbons, P.J., 1993. Temporal factors and speech recognition performance in young and elderly listeners. J Speech Hear Res 36, 1276-1285.

Greenwood, D.D., 1990. A cochlear frequency-position function for several species---29 years later. J. Acoust. Soc. Am. 87, 2592-2605.

Heinrich, A., Carlyon, R.P., Davis, M.H., Johnsrude, I.S., 2008. Illusory vowels resulting from perceptual continuity: a functional magnetic resonance imaging study. J. Cogn. Neurosci. 20, 1737-1752.

Horváth, J., Burgyán, A., 2011. Distraction and the auditory attentional blink. Atten Percept Psychophys 73, 695701.

Huggins, A.W.F., 1964. Distortion of the Temporal Pattern of Speech: Interruption and Alternation. The Journal of the Acoustical Society of America 36, 1055-1064.

Kashino, M., 2006. Phonemic restoration: The brain creates missing speech sounds. Acoust. Sci. \& Tech. 27, 318321.

Kidd, G.R., Humes, L.E., 2012. Effects of age and hearing loss on the recognition of interrupted words in isolation and in sentences. J Acoust Soc Am 131, 1434-1448.

King, A.J., 2007. Auditory neuroscience: filling in the gaps. Curr. Biol. 17, R799-801.

Lister, J., Besing, J., Koehnke, J., 2002. Effects of age and frequency disparity on gap discrimination. J. Acoust. Soc. Am. 111, 2793-2800.

Lister, J., Tarver, K., 2004. Effect of age on silent gap discrimination in synthetic speech stimuli. Journal of Speech, Language and Hearing Research 47, 257.

Loizou, P.C., 1998. Mimicking the human ear. IEEE Signal Processing Magazine 15, 101-130.

Miller, G.A., Licklider, J.C.R., 1950. The Intelligibility of Interrupted Speech. J. Acoust. Soc. Am. 22, 167.

Nelson, P.B., Jin, S.-H., 2004. Factors affecting speech understanding in gated interference: cochlear implant users and normal-hearing listeners. J. Acoust. Soc. Am. 115, 2286-2294.

Nelson, P.B., Jin, S.-H., Carney, A.E., Nelson, D.A., 2003. Understanding speech in modulated interference: cochlear implant users and normal-hearing listeners. J. Acoust. Soc. Am. 113, 961-968.

Nie, K., Barco, A., Zeng, F.-G., 2006. Spectral and temporal cues in cochlear implant speech perception. Ear Hear 27, 208-217.

Petkov, C.I., O’Connor, K.N., Sutter, M.L., 2003. Illusory sound perception in macaque monkeys. The Journal of neuroscience 23, 9155-9161.

Pichora-Fuller, M.K., Schneider, B.A., Benson, N.J., Hamstra, S.J., Storzer, E., 2006. Effect of age on detection of gaps in speech and nonspeech markers varying in duration and spectral symmetry. The Journal of the Acoustical Society of America 119, 1143.

Powers, G.L., Wilcox, J.C., 1977. Intelligibility of temporally interrupted speech with and without intervening noise. J. Acoust. Soc. Am. 61, 195.

Qin, M.K., Oxenham, A.J., 2003. Effects of simulated cochlear-implant processing on speech reception in fluctuating maskers. J. Acoust. Soc. Am. 114, 446-454.

Repp, B.H., 1992. Perceptual restoration of a "missing" speech sound: auditory induction or illusion? Percept Psychophys 51, 14-32.

Repp, B.H., Liberman, A.M., Eccardt, T., Pesetsky, D., 1978. Perceptual integration of acoustic cues for stop, fricative, and affricate manner. J Exp Psychol Hum Percept Perform 4, 621-637.

Riecke, L., Mendelsohn, D., Schreiner, C., Formisano, E., 2009. The continuity illusion adapts to the auditory scene. Hearing Research 247, 71-77.

Riecke, L., Micheyl, C., Vanbussel, M., Schreiner, C.S., Mendelsohn, D., Formisano, E., 2011. Recalibration of the auditory continuity illusion: sensory and decisional effects. Hear. Res. 277, 152-162.

Riecke, L., Vanbussel, M., Hausfeld, L., Baskent, D., Formisano, E., Esposito, F., 2012. Hearing an Illusory Vowel in Noise: Suppression of Auditory Cortical Activity. Journal of Neuroscience 32, 8024-8034.

Rosen, S., 1992. Temporal Information in Speech: Acoustic, Auditory and Linguistic Aspects. Phil. Trans. R. Soc. Lond. B 336, 367-373. 
Saija, J.D., Akyürek, E.G., Andringa, T.C., Başkent, D., 2013. Perceptual Restoration of Degraded Speech Is Preserved with Advancing Age. J. Assoc. Res. Otolaryngol.

Shahin, A.J., Bishop, C.W., Miller, L.M., 2009. Neural mechanisms for illusory filling-in of degraded speech. Neuroimage 44, 1133-1143.

Shannon, R.V., Zeng, F.-G., Kamath, V., Wygonski, J., Ekelid, M., 1995. Speech recognition with primarily temporal cues. Science 270, 303-304.

Shinn-Cunningham, B., Mehraei, G., Bressler, S., Masud, S., 2013. Influences of perceptual continuity on everyday listening. In: ICA 2013 Montreal. ASA, Montreal, Canada, p. 010026.

Shinn-Cunningham, B.G., 2008. Object-based auditory and visual attention. Trends in Cognitive Sciences 12, $182-186$.

Shinn-Cunningham, B.G., Wang, D., 2008. Influences of auditory object formation on phonemic restoration. J. Acoust. Soc. Am. 123, 295-301.

Srinivasan, S., Wang, D., 2005. A schema-based model for phonemic restoration. Speech Communication 45, 6387.

Stephens, D., 1996. Hearing rehabilitation in a psychosocial framework. Scand. Audiol. Suppl. 43, 57-66.

Stickney, G.S., Zeng, F.-G., Litovsky, R., Assmann, P., 2004. Cochlear implant speech recognition with speech maskers. J. Acoust. Soc. Am. 116, 1081-1091.

Stilp, C.E., Goupell, M.J., Kluender, K.R., 2013. Speech perception in simulated electric hearing exploits information-bearing acoustic change. The Journal of the Acoustical Society of America 133, EL136-EL141.

Stilp, C.E., Kluender, K.R., 2010. Cochlea-scaled entropy, not consonants, vowels, or time, best predicts speech intelligibility. PNAS 107, 12387-12392.

Strouse, A., Ashmead, D.H., Ohde, R.N., Grantham, D.W., 1998. Temporal processing in the aging auditory system. J. Acoust. Soc. Am. 104, 2385-2399.

Studebaker, G.A., 1985. A “rationalized” arcsine transform. J. Speech Hear. Res. 28, 455-462.

Sugita, Y., 1997. Neuronal correlates of auditory induction in the cat cortex. Neuroreport 8, 1155-1159.

Tasell, D.J.V., Greenfield, D.G., Logemann, J.J., Nelson, D.A., 1992. Temporal cues for consonant recognition: Training, talker generalization, and use in evaluation of cochlear implants. The Journal of the Acoustical Society of America 92, 1247-1257.

Thurlow, W., 1957. An auditory figure-ground effect. Am. J. Psychol. 70, 653-654.

Thurlow, W.R., Elfner, L.F., 1959. Continuity Effects with Alternately Sounding Tones. The Journal of the Acoustical Society of America 31, 1337-1339.

Verschuure, J., Brocaar, M.P., 1983. Intelligibility of interrupted meaningful and nonsense speech with and without intervening noise. Percept. Psychophys. 33, 232-240.

Versfeld, N.J., Daalder, L., Festen, J.M., Houtgast, T., 2000. Method for the selection of sentence materials for efficient measurement of the speech reception threshold. J. Acoust. Soc. Am. 107, 1671-1684.

Warren, R.M., 1970. Perceptual Restoration of Missing Speech Sounds. Science 167, 392-393.

Warren, R.M., 1984. Perceptual restoration of obliterated sounds. Psychological Bulletin 96, 371-383.

Warren, R.M., Bashford, J.A., Jr, Healy, E.W., Brubaker, B.S., 1994. Auditory induction: reciprocal changes in alternating sounds. Percept Psychophys 55, 313-322.

Warren, R.M., Obusek, C.J., 1971. Speech perception and phonemic restorations. Perception \& Psychophysics 9 , 358-362.

Warren, R.M., Obusek, C.J., Ackroff, J.M., 1972. Auditory induction: perceptual synthesis of absent sounds. Science 176, 1149-1151. 